\title{
Deja Vu, on a different stage
}

\author{
A strange metal in a bosonic system \\ Authors: Chao Yang, Haiwen Liu, Yi Liu, Jiandong Wang, Sishuang Wang, Yang \\ Wang, Qianmei He, Yue Tang, Jian Wang, X.C. Xie, James M. Valles Jr., Jie Xiong, \\ and Yanrong Li \\ arXiv:2105.02654

\section{Recommended with a Commentary by Chandra Varma, University of California, Berkeley and Riverside.}

There has been a wide-spread belief that bosons exist at $T \rightarrow 0$ only either as insulating crystals, or as superfluids. This may be thought of in parallel with the belief that fermions exist at $T \rightarrow 0$ only as either spin-crystals (anti-ferromagnets or ferromagnets of various varieties) or as superconductors. (Disorder may turn the crystals in both cases to be glassy.) The idea of spin-liquids forcefully advocated by P. W. Anderson questions these beliefs. It appears that indeed spin-liquids may be realized at odd integer filling of fermions in a lattice by the combination of quantum-fluctuations and suitably frustrated lattice structures. Much interesting work is being pursued in that direction. But we have had evidence for a number of years now for the existence of a "super-metal" in the low temperature limit in a boson system, in between the superconductor and a boson insulator. It appears too early to draw any parallel to the idea of spin-liquids in fermions - this needs both more experiments and certainly more creative theory.

The experiments on $2 \mathrm{~d}$ metallic films as of about two years ago have been reviewed [1]. The pioneering early experiments [2] revealed that as function of film thickness, the resistivity changed from tending to 0 - the superconducting phase to $\infty$ - the insulating phase with the change occurring close to a resistance per square $h /(2 e)^{2}$ the quantum of resistance with Cooper pair charge. This suggested that the insulator was an insulator of Cooper pairs. Soon models were developed with "duality" - the quantum xy model for superconductivity/superfluidity through freezing of vortices so that conductivity could be infinity, or mobile vortices due to quantum fluctuations so that the conductivity could be zero. Specific predictions about the scaling of conductivity with temperature and electric field were made which appeared successful.

The discovery of a metallic phase at the lowest temperatures in between the insulating and the superconducting phase $[3,1]$ might appear to up-end this neat picture or at least show its incompleteness. The anomalous metallic phase with very high conductivity emerges in the phase diagram as the normal state resistance per square is increased above about $h /(2 e)^{2}$. This is shown in the figure given in the region marked AM for anomalous metal, culled from the paper by Yang et al., highlighted above, based on experiments on a film 

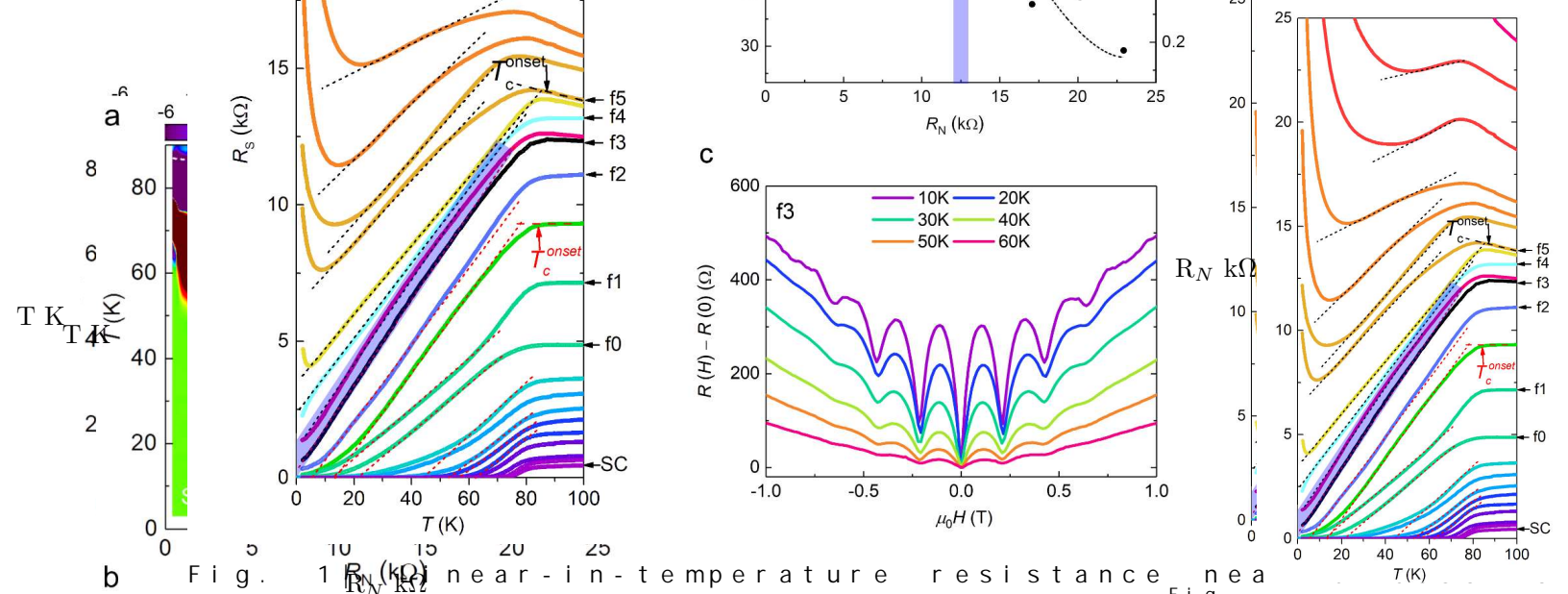

Figure 1: These figures are taken from the highlighted paper by Yang et al. Left panel shows the phase diagram deduced from the low temperature resistivity as the normal state resistance per square is changed. The green region at low temperature at the left has a superconducting ground state. AM is the anomalous metallic phase and "Insulator" is the Cooper pair insulator phase. The region termed "Bosonic strange metal" has resistivity which varies linearly in temperature in the green region as well as linear in magnetic field for a fixed low temperature. The middle panel shows the periodicity of the resistivity with magnetic field applied in sample f3 which has the deepest region of green in the left panel. From the size of the holes dug in the sample the period is estimated to be twice the flux quantum. Similar variation with much smaller amplitude in the region marked insulator, not too far from the region "QCP" may be seen in the paper by Yang et al. The right panel shows the resistivity as a function of temperature for various samples in the green region of the left panel.

of the high temperature superconducting compound $\mathrm{YBa}_{2} \mathrm{Cu}_{3} \mathrm{O}_{6+\delta}$, with holes drilled in it to change the normal state resistance. The critical point appears to exist for normal state resistance of about $h /\left(2 e^{2}\right)$ But how the other parameters in the experiments, the capacitance and the Josephson coupling change across the three zero temperature phases could perhaps be ascertained more adequately.

What might be the basic ingredients of a model which gives such a phase diagram? Aspects of a model with xy superconducting fluctuations in a $2 \mathrm{~d}$ metal and a Cooper pair insulator must be retained because the insulating phase shows magneto-oscillations in resistivity with $2 e$ flux quantization although with vastly reduced amplitude compared to the superconducting phase. This is possible to show in the samples used by Yang et al., because of the nearly periodic array of holes put in them with known dimensions. The amplitude decreases further on going deeper into the insulating phase. The best evidence of that I have seen is in the paper highlighted above and is reproduced here in the figure. In an earlier paper by the same team the oscillations in the resistivity of the anomalous metallic state with the same flux quantization are also shown so that we must think of that phase as also closely related to superconductivity. One may also infer relation to superconductivity also from the hall resistance being so small [1] as to be immeasurable calling for particle-hole sym- 
metry. Further evidence and information on the anomalous metallic state and the scattering mechanism at work is revealed in the broad band micro-wave conductivity measurements [6].

What is truly new and remarkable in the paper above is that the resistivity at the boundary between the insulating and the anomalous metallic phase is linear in temperature. The cross-overs on either side have the shape, shown in the figure, which we have become used to in quantum-critical problems. This is however quite a new context. The by now familiar quantum-criticality in metals, in the cuprates, the anti-ferromagnetic Fe-based superconductors and the heavy fermions is at the critical point of a normal ordered phase and a Fermi-liquid. Now we have linear in T resistivity at low temperatures at what appears to be a quantum-critical point between an anomalous metallic phase with evidence of Cooper pairs and an insulating phase of Cooper pairs. The resistivity per square is very close to $\left(h / 2 e^{2}\right) T / T_{0}$ where $T_{0}$ is the superconducting transition of the film with lowest resistance. Actually, with $T_{0}$ of $60 \mathrm{~K}$, the lowest that the resistivity is linear is only $3 \mathrm{~K}$ but that may be a crossover because the hole density used to tune the resistance cannot be tuned continuously. Equally remarkably, the resistance is also linear in a magnetic field applied perpendicular to the films and to a good approximation to $\left(h / 2 e^{2}\right) H / H_{0}$ with $g \mu_{B} H_{0} \approx k_{B} T_{0}$. We have seen this also in both the cuprates at quantum-criticality to a time-reversal breaking phase and at antiferromagnetic quantum-criticality.

The anomalous metallic phase occurs naturally in a model suggested by Das and Doniach [4] in which the phase of the superconducting order parameter as well as its conjugate, the angular momentum are dynamical variables, which are coupled naturally through their

mutual non-commutation. This model lacks "duality". The ordering of the phase corresponds of-course to the superconducting phase while the ordering of the angular momentum corresponds to a crystallization of vortices giving the insulating phase. In between the two a phase is suggested with high conductivity which may be the anomalous metallic phase. However, the treatment of the model is qualitative, the discussion is based on by now known to be doubious ideas of resonating valence bonds. More work should be done on this model but it also requires augmentation; there does not appear to be any avenue in the model to give linear in $\mathrm{T}$ or linear in $\mathrm{H}$ resistivity.

\section{References}

[1] Aharon Kapitulnik, Steven A. Kivelson, and Boris Spivak, Rev. Mod. Phys. 91, 011002 (2019).

[2] H.M. Jaeger, D. B. Haviland, B. G. Orr, and A. M. Goldman, Phys. Rev. B 40, 182 (1989).

[3] D. A. Ephron, A. Yazdani, A. Kapitulnik, and M. R. Beasley, Phys. Rev. Lett. 76, 1529 (1996).

[4] D. Das and S. Doniach, Phys. Rev. B 60, 1261 (1999).

[5] Yang C. et al., Science 366, 1273 (2007). https://arxiv.org/abs/2006.14115 (2020). 
[6] Y. Wang, I. Tamir, D. Shahar and N.P. Armitage, arXiv:1708.0908. 